\title{
IMPACT OF TECHNICAL INTERVENTION ON PROFITABILITY OF VILLAGE BASED MEDIUM SCALED BROILER ENTERPRISE IN BANGLADESH
}

\author{
J. Jaim and M. N. Islam ${ }^{1}$ \\ Faculty of Business Administration, Eastern University, Dhanmondi, Dhaka, Bangladesh
}

\begin{abstract}
In Bangladesh the majority of the population, particularly the rural people are poverty stricken. This study assesses the possibility of addressing poverty through an advanced technology of broiler rearing model which ensures high participation of rural women, protects the environment and secures from harmful or unnecessary residual effects on human health. Bangladesh Rural Enterprise and Agricultural Project-II (BREAD-II) of Winrock International, a non-profit organization has introduced this model. The present study focuses on the impact of this model on rural household income through comparing profitability of broiler farms adopting this model compared to those of nonadopters. This comparative study confirmed higher profit of BREAD-II Model of broiler rearing which eventually indicates that it can be considered as an effective tool for poverty alleviation.
\end{abstract}

Key words : Broiler enterprise, Vaccination, Drug usages, Bio-security, Profitability

\section{INTRODUCTION}

In the expedition of the economic development of Bangladesh, the policies regarding poverty alleviation have undergone through an array of changes during the last three decades or so. In the 50s and 60s economic growth was regarded as the primary means of poverty alleviation. It was considered that if an economy could ensure a higher growth rate sustaining for a longer period, that growth rate itself would take care of poverty. Afterwards, the emphasis transferred to providing direct provisions for the poor. Whereas the preceding polices were target neutral and indirect in nature, succeeding policies are target oriented and intends to enhance the participation of the poor people in the growth process. In case of direct provision for the poor, the prominence is on meeting the basic needs of the people with particular focus on human resource development, women in development and environment sustainability.

However, the government has accorded the highest priority to the agriculture sector to make this sector economically profitable. Though livestock, one important sub-sector of agriculture is highly a viable sector for generation of employment and income for the landless, unemployed youth and destitute women, little attention was given to this sub-

1 Assistant Professor, Department of Business Administration, Jahangirnagar University, Savar, Dhaka, Bangladesh 
sector until the recent past. In spite of that, from the economic point of view broiler farming is playing a very important role now-a-days in Bangladesh. In fact, broiler has a shorter life cycle and production of broiler requires relatively less capital and land compared to other meat-producing animals. Furthermore, it generates income throughout the year. Besides, broiler enterprise creates various job opportunities for the unemployed people through the establishment of hatchery, feed mills, equipment, manufacturing factories as well as processing and marketing of broilers.

Therefore, there can be a policy of poverty alleviation that introduces an innovative approach in broiler rearing as a means of highly profitable enterprise, ensuring high participation of rural women while protecting the environment without sacrificing the security from harmful or unnecessary residual effects on human health. Such a scheme is introduced by Bangladesh Rural Enterprise and Agricultural Project-II (BREAD-II Project) of Winrock International. With the above background the main objectives of this paper are :

- To provide a synopsis of the technical intervention of the BREAD-II Model of broiler rearing.

- To compare profitability of the farmers with and without intervention of BREADII Model.

\section{METHODOLOGY}

For this study, field level primary data were collected from Singair Upazila of Manikgonj district where BREAD- II Model was tested. Both the BREAD-II Farmers and NonBREAD-II Farmers were from different unions of Singair Upazilla of Manikganj. Out of 24 BREAD-II farms, 20 farms were randomly selected. It may be mentioned here that farms under BREAD-II Model belonged to a partner NGO named as NUSRA. For comparative study, another 20 farms were also selected randomly from a list of NonBREAD-II Farms. Besides, selected poultry farms, other primary sources of data were Livestock Specialist, Winrock International; Thana Livestock Officers; Retailer of Dispensary; Dealer of Chick of Singair, Manikganj. Data were also collected from secondary sources which were mainly Cost-Benefit Analysis of NUSRA and some other publications.

Data related to costs and returns were available from Cost-Benefit Analysis for profit measurement of BREAD-II Farmers. However, BREAD-II Farmers were also interviewed for identifying their problems. In the case of Non-BREAD-II Farms, all information was collected through direct interview using a questionnaire. Most of the questions were close-ended; only few were open-ended. For both types of farms, the last cycle of broiler rearing was completed within April 15 to June 30, 2005 and data related to costs and returns covered this period. 
For the comparison of profitability, Net Return was calculated considering per unit basis (per broiler). For this purpose, the following formula was used:

$$
\Pi=\mathrm{Pa}-\sum \mathrm{Pxi}-\sum \mathrm{Pyi}-\mathrm{Wt}
$$

Where,

$$
\begin{aligned}
\prod_{\mathrm{Pa}} & =\text { Profit per broiler per cycle }(\mathrm{Tk} / \text { cycle) } \\
& =\frac{\sum \text { Unit price of broiler } \mathrm{x} \text { Number of broiler sold and consumed }}{\text { Number of broiler sold and consumed }} \\
\text { Pxi } & =\text { Per broiler unit price of ith (variable) inputs }(\mathrm{i}=1,2,3,--\mathrm{n}) \\
& =\frac{\sum \text { Unit price of variable input } \mathrm{x} \text { Number of input }}{\text { Number of chick purchased }} \\
\text { Pyi } & =\text { Per broiler unit price of ith (depreciation) inputs }(\mathrm{i}=1,2,3,--\mathrm{n}) \\
& =\frac{\sum \text { Depreciation cost of each input }}{\text { Number of chick purchased }} \\
\mathrm{Wt} & =\text { Per broiler wastage including depreciation cost and variable cost } \\
& =\frac{\text { Total fixed and variable cost per broiler } \mathrm{x} \text { Number of dead broiler }}{\text { Number of broiler sold and consumed }}
\end{aligned}
$$

To test statistical significance of the differences of various costs and return items of BREAD II Project and Non-BREAD II Project T-tests were applied. The test statistic is student' $\mathrm{s}-\mathrm{t}$ which is defined as:

$$
\mathrm{t}=\frac{\overline{\mathrm{X}}_{1}-\overline{\mathrm{X}}_{2}}{\sqrt{\frac{\mathrm{s}_{1}^{2}}{\mathrm{n}_{1}}+\frac{\mathrm{s}_{2}{ }^{2}}{\mathrm{n}_{2}}}}
$$

with degrees of freedom $\left(\mathrm{n}_{1+} \mathrm{n}_{2}-2\right)$

Where,

$\overline{\mathrm{X}}_{1}, \overline{\mathrm{X}}_{2}$ are the sample means

$\mathrm{S}_{1}{ }^{2}$ and $\mathrm{S}_{2}{ }^{2}$ are corresponding variances and

$\mathrm{n}_{1}$ and $\mathrm{n}_{2}$ are the sample sizes.

A few limitations of analysis of this study may be noted as follows:

- Though the BREAD-II Farmers keep actual record of data regarding financial activities and other broiler rearing activities such as weight of broilers, the 
Non-BREAD-II Farmers did not perform this practice. Thus, as the data was not so reliable for the Non-BREAD-II Farmers, the comparison is not accurate.

- As the litter had not yet been sold and the market of it was not so structured, the income from litter was excluded from the analysis.

- The opportunity cost of labour was ignored as family labour was engaged in poultry farms that were basically unemployed.

- As the comparison is based on the last cycle of broiler rearing, the time range is encountered as two and half month. Due to such lengthy period, price fluctuation of broiler took place that eventually has impact on overall analysis.

- BREAD-II Farmers enjoy economies of scale in many cases such as vaccination, transportation etc. while Non-BREAD-II Farmers enjoy economies of scale in the case of fixed costs as the number of broilers is larger for many of them. But here, the economies of scale have been ignored.

- For the simplicity of the study, in case of measuring Wastage, the cost is determined on the basis of total unit cost instead of allocating cost on average lifetime of dead broiler.

\section{OVERVIEW ON BREAD-II PROJECT OF WINROCK INTERNATIONAL}

\section{Background of the project}

Winrock International (WI), a non-profit international organization launched Bangladesh Rural Enterprise and Agricultural Project-II (BREAD-II) with effect from June, 2003. This three-year project is funded by United States Department of Agriculture (USDA). The mission of the project is to alleviate poverty and increase food security through poultry and maize production, NGO-implemented agriculture and enterprise development programs and training for farmers and trainers in agribusiness and capacity building. The BREAD-II Project supports poultry production by the rural poor in two ways:

○ Directly through promotion of medium-scale village poultry enterprises for small-holders;

- Indirectly through promotion of maize production to lower the cost of poultry feed.

\section{Overview on BREAD-II poultry program}

BREAD-II project addresses the opportunity of medium-scale commercialization, for small-holders to manage 100-500 birds to produce a uniform product of acceptable quality. Consideration of uniformity and product quality is necessary to assure a regular and substantial return on investment, with a minimum of risk.

Target beneficiaries are rural families in the small-holder category (0.5-1.0 ha of land). Being close to the homestead, this type of enterprise is ideal for high levels of participation of women, with consequent benefits to the entire household. Winrock International undertook a comprehensive training program to develop the capacity of the 
NGO partners and the farm beneficiaries to address complex technical requirements for promoting profitable medium scale poultry enterprises in the rural areas.

At present, five Partner NGOs have been selected and awarded Sub-Grant Agreements for the BREAD-II poultry program operating in Sherpur, Manikganj and Gazipur. Through these NGOs, all costs, except housing for the broiler rearing farm is covered by the project. However, after successful implementation of this farm, individual beneficiaries pay back actual investment to the partner NGO. Partner NGO then reinvest this fund for further farming. Trained NGO staff technically support the farmers with support from the BREAD-II poultry expert. Low cost options, without unduly jeopardizing product quality and uniformity requirements, are sought so as to minimize investment requirements that eventually enhances prospects of wider adoption.

\section{Specialty of broiler rearing by BREAD-II}

\section{Distinctive features of broiler rearing practice}

- Distinctiveness in vaccine schedule: According to Winrock International, the Day Old Chick of broiler is provided with the killed vaccine - Gamborifa vaccine for the prevention of Gamboro disease, one of the most devastating diseases of broiler. But in general, this killed vaccine is not provided to the chicks on the first day by the farmers in our country.

- Distinctiveness in drug usage: Due to the residual effect on human health some of the poultry medicines and growth promoters are banned in many countries such as Tetracycline, Furazolidon, Chlorumphenicol etc. But in Bangladesh, some of these medicines are widely used not only after dictating diseases, but also in general, as a routine. It should be mentioned that these medicines and growth promoters are not banned in our country. Winrock International does not allow premeditation and does not use these banned medicines for their farming. Rather it tries to have less dependency on drug.

\section{Other specialty of broiler rearing practice of BREAD-II}

- Broiler rearing on stage: Instead of using floor, the practice of rearing broilers on bamboo slat is exercised for hygiene factors. The bamboo slat is fixed one meter above ground and a gangway is constructed at the entrance for easy access to broilers.

- Bio-security for broiler rearing: Enforcement of bio-security arrangements by BREAD-II includes maintaining batches of birds of the same age only, exclusion of vermin, other free-roaming poultry or birds and animals, quarantine procedures for humans (foot-baths at entrance, no casual visitors), adequate waste and dead bird disposal, disaffection capability, use of disease-free chicks and feed etc. 


\section{COMPARATIVE PROFITABILITY ANALYSIS WITH AND WITHOUT INTERVENTION OF WINROCK INTERNATIONAL}

\section{Comparative analysis on fixed cost}

There is no significant difference in fixed cost (total depreciation cost) between BREAD-II Farmers (Tk. 2.64) and Non-BREAD-II Farmers (Tk. 2.60) (Annexure Table 1). But in the case of shed building and repairing cost the BREAD-II Farmers are cost efficient. BREADII Farmers use comparatively cheap materials to build sheds. On the other hand, in general, Non-BREAD-II Farmers build sheds with comparatively high priced materials such as tin.

Excluding shed building and repairing cost, BREAD-II Farmers are incurring more fixed cost and the significant cost difference is for brooder and brooder guard, feeder and other fixed assets. Moreover, no Non-BREAD-II Farmer uses conceal pit (costing Tk. 1,500) which is a must for bio-security and thermometer (costing Tk. 400) for assessing the healthy condition of broilers. At present, these costs are justified for ensuring quality broiler as well as maintaining healthy environmental.

Compared to Non-BREAD-II Farmers, BREAD-II Farmers are incurring significantly low variable cost as can be seen from Table 1. Item wise some important variable cost items have been discussed in this section.

Feed: For feed BREAD-II Farmers incur significantly less amount than the Non-BREAD-II Farmers by $27.12 \%$ (Tk. 10.15) per broiler. In fact, the feed consumption per broiler of Non-BREAD-II Farmers $(2.80 \mathrm{~kg})$ is higher than that of BREAD-II Farmers $(2.26 \mathrm{~kg})$ by $23.89 \%$. But as the average weight of two types of broiler is different and amount of feed consumption is related with gaining weight of broiler, it is better to analyze the feed cost considering feed consumption and weight of broiler.

Table 1. Variable cost per broiler

\begin{tabular}{|c|c|c|c|c|c|}
\hline \multirow{2}{*}{$\begin{array}{c}\text { Cost of different } \\
\text { items }\end{array}$} & \multicolumn{2}{|c|}{ BREAD-II farms } & \multicolumn{2}{|c|}{ Non-BREAD-II farms } & \multirow{2}{*}{$\begin{array}{c}\text { Difference of costs } \\
\text { (BREAD-II - } \\
\text { Non-BREAD-II) }\end{array}$} \\
\hline & \begin{tabular}{|l} 
Actual cost \\
(Tk.)
\end{tabular} & $\begin{array}{c}\% \text { of total } \\
\text { cost }\end{array}$ & \begin{tabular}{|c|} 
Actual cost \\
(Tk.)
\end{tabular} & $\begin{array}{c}\% \text { of total } \\
\text { cost }\end{array}$ & \\
\hline Chick & 23.30 & 34.13 & 22.75 & 28.64 & 0.55 \\
\hline Feed & 37.43 & 54.83 & 47.58 & 59.90 & $-10.15^{*}$ \\
\hline Vaccination & 2.67 & 3.91 & 1.59 & 2.00 & $1.08^{*}$ \\
\hline Medicine & 0.37 & 0.54 & 4.62 & 5.82 & $-4.25^{*}$ \\
\hline Transportation & 1.51 & 2.21 & 1.04 & 1.31 & $0.47^{*}$ \\
\hline Veterinary service & 0.85 & 1.25 & 0.00 & 0.00 & $0.85^{*}$ \\
\hline Husk & 1.10 & 1.61 & 0.44 & 0.55 & $0.66^{*}$ \\
\hline Disinfectant & 0.55 & 0.81 & 0.88 & 1.11 & -0.33 \\
\hline Water chlorination & 0.23 & 0.34 & 0.11 & 0.14 & 0.12 \\
\hline Electricity & 0.25 & 0.37 & 0.34 & 0.43 & -0.09 \\
\hline Others & 0.00 & 0.00 & 0.08 & 0.10 & $-0.08^{*}$ \\
\hline Total variable cost per bird & 68.26 & 100 & 79.43 & 100 & $-11.15^{*}$ \\
\hline
\end{tabular}


For BREAD-II Farms feed consumption per Kg broiler is $13.50 \%$ lower than that of the Non-BREAD-II Farms. Average price per Kg feed is also lower for BREAD-II Farms. As a result, average cost of feed per Kg broiler is higher for Non-BREAD-II Farms by $15.60 \%$. It may be mentioned here that all BREAD-II Farmers use "Nourish" feed, whereas only 15\% Non-BREAD-II Farmers use "Nourish" feed. Though "Nourish" is comparatively cheap, at present it is the best poultry feed according to the research findings of Nutrition Division, Department of Livestock, Bangladesh (July, 2005).

Table 2. Feed consumption per kg broiler

\begin{tabular}{lcc}
\hline \multicolumn{1}{c|}{ Particulars } & BREAD-II Farms & Non-BREAD-II Farms \\
\hline Feed consumption per kg broiler & 1.73 & 2.00 \\
Average price per kg feed & 16.60 & 16.77 \\
Average cost of feed per kg broiler & 28.72 & 33.20 \\
\hline
\end{tabular}

Source : Cost-benefit analysis, NUSRA, November, 2004 - July, 2005 and Field survey

Vaccination: Non-BREAD-II Farmers incur less cost regarding vaccination cost by $67.92 \%$. According to Thana Livestock Office, the general perception of the farmers of the area is to have the risk of diseases of broiler rather than initially reimburse money for vaccine.

However, all Non-BREAD-II Farmers use the first dose of BCRDV vaccine for preventing New Castle Disease. Only 55\% farmers use Gamboro vaccine. All these vaccines are of low priced and are not properly maintained in cool condition. Moreover, no NonBREAD-II Farmers use Gamborifa or Barsa Plex or Barsine Plus vaccines that are used for BREAD-II Farms in order to prevent Gamboro.

Medicine: The cost of medicine (Tk. 0.37 per broiler) is negligible $(0.01 \%)$ considering the total cost for BREAD-II Farmers. But for Non-BREAD-II Farmers, it is the third highest cost $(5.82 \%$ of the total cost) that incurs Tk. 4.62 per broiler. Therefore, Non-BREAD-II Farmers spend 12.49 times more than BREAD-II Farmers for medicine. However, 35\% BREAD-II Farms did not require any medicine and the highest cost is only Tk. 3.23 per broiler. On the other hand, Non-BREAD-II Farmers spend at least Tk. 2.00 and maximum Tk. 8.00 per broiler for medicine.

In fact, BREAD-II Farmers use medicine and vitamin, mineral only when it is necessary. But Non-BREAD-II Farmers use many of these on a routine basis. Moreover, the medicine and vitamin which are banned in many countries are widely used by Non-BREAD-II Farmers.

Transportation: The cost of transportation includes cost for transporting chick and feed. All Non-BREAD-II Farmers use van for this purpose and generally they purchase chick and feed from Singairbazar, Maniklganj. But for BREAD-II Farmers chicks are purchased from the hatchery - "Nourish" of Gazipur and for the mode of transport, pickup is used. 
Along with chick, feed is also transported. Then the cost is shared by each farmer. It should be mentioned that at present, there is no dealer of "Nourish" at Singair and the feed "Nourish" is not available there.

Veterinary service: BREAD-II Farmers incur significantly high cost for veterinary service. The reason is that no Non-BREAD-II Farmers take veterinary service for broilers. From own experience or taking suggestion from other farmers or dispensary, they go for the treatment.

Husk and other variable costs: Husk cost is significantly high for BREAD-II Farmers and other variable cost is significantly high for Non-BREAD-II Farmers. But, considering total cost these are minor costs. Further, it may be noted here that although the BREAD-II Farmers are incurring significantly more cost for vaccination, transportation, veterinary service and husk; for ensuring quality product and considering environmental aspect, in general there is no justified scope to minimize these costs.

\section{Comparative analysis on wastage}

The average cost of wastage per broiler of BREAD-II Farms (Tk. 2.97) is 50.51\% less than that of Non-BREAD-II Farmers (Tk. 4.47). In fact, this cost basically depends on the number of dead broilers. As the average mortality rate of broiler is less for BREAD-II Farms $(3.88 \%)$ than that of Non-BREAD-II Farms (4.79\%) this eventually reduces cost.

\section{Comparative analysis on pricing}

It has been found that there is no significant difference in price per $\mathrm{Kg}$. of broiler of the two types of farms (Table 3). But average price per broiler of BREAD-II Farms is significantly less than that of Non-BREAD-II Farms because of the difference in weight of broilers.

Table 3. Broiler Pricing

(In Taka)

\begin{tabular}{l|c|c|c}
\hline \multicolumn{1}{c|}{ Particulars } & $\begin{array}{c}\text { BREAD-II } \\
\text { farms }\end{array}$ & $\begin{array}{c}\text { Non-BREAD-II } \\
\text { farms }\end{array}$ & $\begin{array}{c}\text { Price difference } \\
\text { (BREAD-II - Non-BREAD-II) }\end{array}$ \\
\hline Average Rate per Kg Broiler & 60.62 & 61.49 & -0.87 \\
Average Price per Broiler & 83.05 & 91.50 & $-8.45^{*}$ \\
\hline
\end{tabular}

Note : * Significant at $5 \%$ level

Source : Cost-benefit analysis, NUSRA, November, 2004 - July, 2005 and Field survey

\section{Comparative analysis of net return}

The analysis shows (Table 4) that Net Return of BREAD-II Farms (Tk. 9.18) is higher than that of the Non-BREAD-II Farms (Tk. 5.01) by $83.23 \%$. For BREAD-II Farms, the Net Return is $12.43 \%$ of the total cost while for Non-BREAD-II Farms, it is $5.79 \%$ of the total cost. 
As the trade is based on the weight of broiler, it is better to analyze the Net Return on the basis of per $\mathrm{Kg}$ broiler. The Net Return per $\mathrm{Kg}$ broiler of BREAD-II Farms is almost double than that of Non-BREAD-II Farms. (Annexure Table 2).

Table 4. Net return per broiler

(In Tk.)

\begin{tabular}{l|c|c}
\hline \multicolumn{1}{c|}{ Particulars } & BREAD-II & Non-BREAD-II \\
\hline Price of broiler sold and consumed & 83.05 & 91.50 \\
Total fixed cost (depreciation cost) & 2.64 & 2.60 \\
Total variable cost & 68.27 & 79.42 \\
Total fixed and variable cost & 70.90 & 82.02 \\
Wastage & 2.97 & 4.47 \\
Total cost (iv +v) & 73.87 & 86.49 \\
Net return per broiler (i - vi) & 9.18 & 5.01 \\
\hline
\end{tabular}

Source : Cost-benefit analysis, NUSRA, November, 2004 - July, 2005 and Field survey

\section{Comparative analysis of net return considering same weight and same price}

Net return of broiler enterprise largely depends on weight of the broiler as well as price of broiler at which it is sold. As the weight as well as price varied in BREAD -II Farms and Non BREAD - II Farms, sensitivity analysis was done to see what happens to the net return if the weight per broiler and price remain same for both types of farms.

\section{Net return at the same weight}

The average weight of broiler of Non-BREAD-II Farms $(1.48 \mathrm{Kg})$ was found significantly higher than that of BREAD-II Farms $(1.36 \mathrm{Kg})$. But not all the Non-BREAD-II Farmers keep record of appropriate weight of broiler. And according to the Thana Livestock Officers, wholesalers, retailers and even the Non-BREAD-II Farmers the weight of broilers of BREAD-II Farmers is much higher than that of the Non-BREAD-II Farmers. But as the total return is calculated based on total weight, the average weight mentioned by Non-BREAD-II Farms eventually has impact on Net Return. To mitigate this limitation, it has been assumed that both types of farms have the average weight of broiler of BREAD-II Farm $(1.36 \mathrm{Kg})$. At the same weight of broiler, the BREAD-II Farms cut a good figure of average Net Return (Tk. 9.18) whereas the Non-BREAD-II Farms incur loss (Tk. 2.17) as can be seen from Table 5.

Table 5. Net return per broiler at the same weight

(In Taka)

\begin{tabular}{l|c|c}
\hline \multicolumn{1}{c|}{ Particulars } & BREAD-II & Non-BREAD-II \\
\hline Price of broiler sold and consumed & 83.05 & 84.32 \\
Total cost & 73.87 & 86.49 \\
Net return & 9.18 & -2.17 \\
\hline
\end{tabular}

Source : Cost-benefit analysis, NUSRA, November, 2004 - July, 2005 and Field survey 


\section{Net return at the same price}

As the price of broiler is highly fluctuating and broilers were sold in a scattered manner at different times within two and half month time horizon, profitability has been reexamined assuming same price for both type of farms. That is average price of Tk. 61.55 for all broilers have been considered assuming that there is no difference in marketing efficiency. At the same price, it has been found that Average Net Return per broiler of BREAD-II Farms is almost double than that of Non-BREAD-II Farms (Table 6).

Table 6. Net return per broiler at the same price

(In Taka)

\begin{tabular}{l|c|c}
\hline \multicolumn{1}{c|}{ Particulars } & BREAD-II & Non-BREAD-II \\
\hline Return per Broiler Sold and Consumed & 84.49 & 91.52 \\
Total Cost & 73.87 & 86.49 \\
Net Return & 10.62 & 5.03 \\
\hline
\end{tabular}

Source : Cost-benefit analysis, NUSRA, November, 2004 - July, 2005 and Field survey

\section{CONCLUSIONS}

The Poultry Promotion Program, operating through the partner NGO, NUSRA at Manikgonj, demonstrates the promising sign of profitability of broiler business comparing with other farms without the intervention of Winrock International. Per broiler, net return of BREAD-II Farms is higher than that of the Non-BREAD-II Farms by $83.23 \%$ and per $\mathrm{Kg}$ broiler, it is almost double for BREAD-II Farms than that of NonBREAD-II Farms. Besides, comparing with Non-BREAD-II Farmers, BREAD-II Farmers are cost efficient. Major cost reduction is due to the feed and medicine cost. On the other hand, BREAD-II Farmers incur more cost for the fixed assets as well as for vaccine, veterinary service and so on. But in general, to ensure the quality of broilers along with considering the environmental aspects, these costs are justified. The overall findings of this study indicates that technical intervention of BREAD-II Model of the Winrock International can make a significant contribution in alleviating rural poverty by enhancing profitability of village based medium scaled broiler enterprise.

\section{REFERENCES}

BREAD-II, Winrock International of Agricultural Development. 2003. 2003 Inception Report.

BREAD-II Winrock International of Agricultural Development. 2004. June, 2003 - May 2004, Annual Report.

NUSRA 2005. November, 2004 - July, 2005 Cost-Benefit Analysis Report of Broiler.

Jaim, J. 2005. Profitability Analysis on Village Based Medium Size Broiler Enterprise: A Study on BREAD-II Project, Winrock International, (Internship Report, Department of Business Administration, Jahangirnagar University, Savar, Dhaka, 2005).

Rahman, M. H. 2004. Medium Scale Commercial Broiler and Layer Production Manual, [Manual].

BREAD-II Project, Winrock International, 2004. Training Material of Broiler Enterprise, [Brochure]. 


\section{ANNEXURE}

Table 1. Depreciation cost and repairing cost per broiler

(In Tk.)

\begin{tabular}{l|c|c|c}
\hline \multicolumn{1}{c|}{ Particulars } & $\begin{array}{c}\text { BREAD-II } \\
\text { farms }\end{array}$ & $\begin{array}{c}\text { Non-BREAD-II } \\
\text { farms }\end{array}$ & $\begin{array}{c}\text { Difference of costs } \\
\text { (BREAD-II - } \\
\text { Non-BREAD-II) }\end{array}$ \\
\hline $\begin{array}{l}\text { Depreciation expense of shed } \\
\text { Repairing cost }\end{array}$ & 0.00 & 1.50 & $-0.20^{*}$ \\
$\begin{array}{l}\text { Depreciation expense of brooder and } \\
\text { brooder guard }\end{array}$ & 0.17 & 0.47 & $-0.47^{*}$ \\
Depreciation expense of feeder & 0.08 & 0.02 & $0.15^{*}$ \\
Depreciation expense of drinker & 0.06 & 0.04 & $0.03^{*}$ \\
Depreciation expense of fan & 0.08 & 0.04 & 0.02 \\
Depreciation expense of other fixed cost ** & 0.75 & 0.27 & 0.04 \\
Total depreciation cost and repairing cost & 2.64 & 2.60 & $0.48^{*}$ \\
\hline
\end{tabular}

Note : * Significant at $5 \%$ level, ${ }^{* *}$ Other fixed cost includes conceal pit, thermometer, jute-sack, polythene, bulb etc.

Source: Cost-benefit analysis, NUSRA, November 2004 - July 2005 and Field survey

Table 2. Net return per kg broiler

(In Tk.)

\begin{tabular}{l|c|c}
\hline \multicolumn{1}{c|}{ Particulars } & BREAD-II & Non-BREAD-II \\
\hline Price of broiler sold and consumed & 61.48 & 61.95 \\
Total fixed (depreciation) cost & 1.97 & 1.77 \\
Total variable cost & 50.58 & 53.98 \\
Total fixed and variable cost & 52.55 & 55.75 \\
Wastage & 2.15 & 3.08 \\
Total cost & 54.70 & 58.83 \\
Net return per kg broiler & 6.79 & 3.12 \\
\hline
\end{tabular}

Source: Cost-benefit analysis, NUSRA, November, 2004 - July, 2005 and field survey 